\title{
Vestibular Migraine
}

\author{
Thomas Lempert, $\mathrm{MD}^{1,2}$ \\ ${ }^{1}$ Department of Neurology, Schlosspark-Klinik, Berlin, Germany \\ ${ }^{2}$ Vestibular Research Group, Charité, Berlin, Germany \\ Semin Neurol 2013;33:212-218.
}

\begin{abstract}
Address for correspondence Thomas Lempert, MD, Neurologische Abteilung, Schlosspark-Klinik, Heubnerweg 2, 14059 Berlin, Germany (e-mail: thomas.lempert@schlosspark-klinik.de).
\end{abstract}

\begin{abstract}
Vestibular migraine presents with attacks of spontaneous or positional vertigo, head motion-induced vertigo, and visual vertigo lasting 5 minutes to 3 days. The recent classification of vestibular migraine, jointly proposed by the Bárány Society and the International Headache Society, allows identification of vestibular migraine and probable vestibular migraine based on explicit criteria. The diagnosis is based on symptom type, severity and duration, a history of migraine, temporal association of migraine symptoms with vertigo attacks, and exclusion of other causes. Because headache is often absent during acute attacks, other migraine features such as photophobia or auras have to be specifically inquired about. During acute attacks, one may find central spontaneous or positional nystagmus, and less commonly, unilateral vestibular hypo-

\section{Keywords}

- migraine

- vestibular

- vertigo

- dizziness function. In the symptom-free interval, vestibular testing adds little to the diagnosis as findings are mostly minor and nonspecific. The pathophysiology of vestibular migraine is unknown, but several mechanisms link the trigeminal system, which is activated during migraine attacks, and the vestibular system. Treatment includes antiemetics for severe acute attacks, pharmacological migraine prophylaxis, and lifestyle changes.
\end{abstract}

When a new disorder enters the medical world, it is usually invented by the pharmaceutical industry trying to expand their markets. The field of neuro-otology may be an exception to this rule, as the last three decades have seen the emergence of new vestibular syndromes on the basis of clinical, epidemiological, and pathophysiological findings, including various subtypes of benign paroxysmal positional vertigo, superior canal dehiscence and-of particular interest herevestibular migraine.

That migraine may present with attacks of vertigo has been repeatedly documented from the early days of neurology. ${ }^{1}$ Starting with Kayan and Hood's classical article, ${ }^{2}$ the clinical features of vestibular migraine have been well elucidated in several large case series. ${ }^{3-9}$ Various terms have been used to designate vertigo caused by a migraine mechanism including migraine-associated vertigo, migraine-associated dizziness, migraine-related vestibulopathy, migrainous vertigo, benign recurrent vertigo, and basilar migraine. The term vestibular migraine has been convincingly advocated as a condition that stresses the particular vestibular manifestation of migraine and thus best avoids confounding with nonvestibular dizziness associated with migraine. ${ }^{10}$ Therefore, the Bárány Society and the International Headache Society (IHS) have opted for vestibular migraine in their recent joint article on the classification of the disorder. ${ }^{11}$ The term basilar-type migraine should be restricted to patients who fulfill the respective diagnostic criteria of the International Classification of Headache Disorders (ICHD). ${ }^{12}$

\section{Epidemiological Aspects}

The recognition of a causal link between vertigo and migraine was promoted by epidemiological observations indicating a more than chance association of migraine with vertigo and dizziness. Dizziness and vertigo rank among the most common complaints in the general population and are frequently reported by patients with migraine. However, the prevalence of migraine has been shown to be elevated among patients with dizziness, ${ }^{8}$ and in particular among patients with unclassified recurrent vertigo. ${ }^{13,14}$ Conversely, significantly more patients with migraine reported vertigo compared with patients with tension-type headache, namely $27 \%$ versus
Copyright (c) 2013 by Thieme Medical Publishers, Inc., 333 Seventh Avenue, New York, NY 10001, USA. Tel: +1(212) 584-4662.
DOI http://dx.doi.org/ 10.1055/s-0033-1354596. ISSN 0271-8235. 
$8 \%{ }^{2}$ Vertigo was also more common in migraine patients than in headache-free controls in two case-control studies. ${ }^{15,16}$ In the general population, migraine headaches and vertigo concur about three times more often than expected by chance. As migraine has a lifetime prevalence of $14 \%{ }^{17}$ and vestibular vertigo of $7 \%,{ }^{18}$ chance concurrence is expected in $1 \%$ of the population, but was actually found in $3.2 \%$ in a large, population-based study. ${ }^{19}$ In individual patients with vertigo and migraine, clinicians must determine whether they have vestibular migraine, that is vertigo that is caused by migraine, or rather dizziness/vertigo of an unrelated cause, which may occur by chance in a migraine patient, or which may be due to one of several dizziness syndromes that are statistically associated with migraine.

\section{Diagnostic Criteria for Vestibular Migraine}

In the current International Classification of headache Disorders (ICHD-2), vertigo is not included as a migraine symptom in adults except in the framework of basilar-type migraine, ${ }^{12}$ which leads to vertigo in more than $60 \%$ of the patients. $^{20}$ As an aura symptom of basilar-type migraine, vertigo should last between 5 and 60 minutes and should be followed by migraine headaches. In addition, at least one more aura symptom from the posterior circulation is required. Less than $10 \%$ of patients with vestibular migraine fulfill the criteria for basilar-type migraine, ${ }^{5-8}$ which makes basilar-type migraine an inappropriate category for most of these patients.

Recently, the Bárány Society, which represents the international community of basic scientists, otolaryngologists, and neurologists committed to vestibular research, mandated a classification group to develop diagnostic criteria for vestibular migraine. The draft of the classification was extensively discussed with the Migraine Classification Committee of the International Headache Society, which resulted in a joint document defining vestibular migraine and probable vestibular migraine. ${ }^{11}$ These criteria will be included in the third edition of the International Classification of Headache Disorders, expected to be published in late 2013, where it will appear in the appendix for new disorders that need further research for validation. In addition, the classification of vestibular migraine is part of the evolving Classification of Vestibular Disorders of the Bárány Society. The new ICHD-3 will only include vestibular migraine, while the Bárány classification will also contain probable vestibular migraine (-Table 1).

\section{Prevalence of Vestibular Migraine}

The prevalence of vestibular migraine was $7 \%$ in a group of 200 consecutive dizziness clinic patients and 9\% in a group of 200 migraine clinic patients. ${ }^{8}$ In a two-stage populationbased study ( $n=4869$ adults) with screening interviews followed by expert telephone interviews, the lifetime prevalence of vestibular migraine was estimated at $0.98 \%$ (95\% confidence interval $0.7-1.37) .{ }^{19}$ of note, vestibular migraine accounted for only a third of migraine patients with a history of vertigo, which indicates the need for a thorough neurootological workup for exclusion of other diagnoses. ${ }^{19}$ In a community-based sample of middle-aged women in Taiwan, vestibular migraine was identified in $5 \%$ and in $30 \%$ of those with migraine. ${ }^{24}$ Vestibular migraine is still widely underdiagnosed, as shown by a study from a dizziness clinic in Switzerland where vestibular migraine accounted for $20.2 \%$ of the diagnoses in young patients, but was suspected by the referring doctors in only $1.8 \%{ }^{25}$

\section{Clinical Features}

\section{Demographic Aspects}

Vestibular migraine may occur at any age. ${ }^{4,5,7}$ It has a female preponderance with a reported female to male ratio between 1.5 and 5 to $1 .^{5-8}$ Familial occurrence is not uncommon, probably based on an autosomal dominant pattern of inheritance with decreased penetrance in men. ${ }^{26}$ In most patients, migraine begins earlier in life than vestibular migraine., ${ }^{7,8}$ Some patients have been free from migraine attacks for years when vestibular migraine first manifests itself. ${ }^{7}$ Not infrequently, migraine headaches are replaced by vertigo attacks in women around menopause.

\section{Vestibular Migraine in Children}

Benign paroxysmal vertigo of childhood is an early manifestation of vestibular migraine that is recognized by the ICHD-2. It is characterized by brief attacks of vertigo or disequilibrium, anxiety, and often nystagmus or vomiting, recurring for months or years in otherwise healthy young children. ${ }^{27}$ Many of these children later develop migraine, often years after vertigo attacks have ceased. ${ }^{28}$ A family history of migraine in first-degree relatives is increased twofold compared with controls. ${ }^{29}$ In a population-based study, the prevalence of recurrent vertigo probably related to migraine was estimated at $2.8 \%$ in children between 6 and 12 years of age. $^{29}$

\section{Clinical Presentation in Adults}

Patients with vestibular migraine typically report spontaneous or positional vertigo. Some experience a sequence of spontaneous vertigo transforming into positional vertigo after several hours or days. ${ }^{30}$ This positional vertigo is distinct from benign paroxysmal positional vertigo (BPPV) with regard to duration of individual attacks (often as long as the head position is maintained in vestibular migraine vs seconds only in BPPV), duration of symptomatic episodes (minutes to days in vestibular migraine vs weeks in BPPV) and nystagmus findings. ${ }^{31}$ Altogether, 40 to $70 \%$ of patients experience positional vertigo in the course of the disease, but not necessarily with every attack..$^{2,26,31}$ A frequent additional symptom is head motion intolerance, i.e., imbalance, illusory motion, and nausea aggravated or provoked by head movements. ${ }^{5,15}$ Visually induced vertigo, that is vertigo provoked by moving visual scenes such as traffic or movies, can be another prominent feature of vestibular migraine. ${ }^{5,32}$ Nausea and imbalance are frequent, but nonspecific accompaniments of acute vestibular migraine. 
Table 1 Diagnostic criteria for vestibular migraine

\begin{tabular}{|c|c|}
\hline & 1. Vestibular migraine \\
\hline A. & At least 5 episodes with vestibular symptoms ${ }^{\mathrm{a}}$ of moderate or severe intensity, ${ }^{\mathrm{b}}$ lasting 5 min to $72 \mathrm{~h}^{\mathrm{c}}$ \\
\hline B. & $\begin{array}{l}\text { Current or previous history of migraine with or without aura according to the International Classification of } \\
\text { Headache Disorders (ICHD) }\end{array}$ \\
\hline C. & $\begin{array}{l}\text { One or more migraine features with at least } 50 \% \text { of the vestibular episodes }{ }^{\mathrm{e}} \text { : } \\
\text { - Headache with at least two of the following characteristics: one-sided location, pulsating quality, moderate or } \\
\text { severe pain intensity, aggravation by routine physical activity } \\
\text { - Photophobia and phonophobia } \\
\text { - Visual aurag }\end{array}$ \\
\hline \multirow[t]{2}{*}{ D. } & Not better accounted for by another vestibular or ICHD diagnosis ${ }^{\mathrm{h}}$ \\
\hline & 2. Probable vestibular migraine \\
\hline A. & At least 5 episodes with vestibular symptoms ${ }^{\mathrm{a}}$ of moderate or severe intensity ${ }^{\mathrm{b}}$, lasting $5 \mathrm{~min}$ to $72 \mathrm{~h}^{\mathrm{c}}$ \\
\hline B. & $\begin{array}{l}\text { Only one of the Criteria B and C for vestibular migraine is fulfilled (migraine history or migraine features during } \\
\text { the episode) }\end{array}$ \\
\hline C. & Not better accounted for by another vestibular or ICHD diagnosis ${ }^{\mathrm{h}}$ \\
\hline
\end{tabular}

${ }^{a}$ Vestibular symptoms, as defined by the Bárány Society's Classification of Vestibular Symptoms (Bisdorff et al ${ }^{21}$ ) and qualifying for a diagnosis of vestibular migraine, include

- Spontaneous vertigo including

- Internal vertigo, a false sensation of self-motion

- External vertigo, a false sensation that the visual surround is spinning or flowing

- Positional vertigo, occurring after a change of head position

- Visually induced vertigo, triggered by a complex or large moving visual stimulus

- Head motion-induced vertigo, occurring during head motion

- Head motion-induced dizziness with nausea. Dizziness is characterized by a sensation of disturbed spatial orientation. Other forms of dizziness are currently not included in the classification of vestibular migraine.

"Vestibular symptoms are rated "moderate" when they interfere with but do not prohibit daily activities and "severe" if daily activities cannot be continued.

'Duration of episodes is highly variable: About $30 \%$ of patients have episodes lasting minutes, $30 \%$ have attacks for hours and another $30 \%$ have attacks over several days. The remaining $10 \%$ have attacks lasting seconds only, which tend to occur repeatedly during head motion, visual stimulation, or after changes of head position. In these patients, episode duration is defined as the total period during which short attacks recur. At the other end of the spectrum, there are patients who may take 4 weeks to fully recover from an episode. However, the core episode rarely exceeds 72 hours. ${ }^{4-8,22}$ ${ }^{\mathrm{d}}$ Migraine categories 1.1 and 1.2 of the ICDH-2. ${ }^{12}$

e One symptom is sufficient during a single episode. Different symptoms may occur during different episodes. Associated symptoms may occur before, during, or after the vestibular symptoms.

${ }^{f}$ Phonophobia is defined as sound-induced discomfort. It is a transient and bilateral phenomenon that must be differentiated from recruitment, which is often unilateral and persistent. Recruitment leads to an enhanced perception and often distortion of loud sounds in an ear with decreased hearing. ${ }^{9}$ Visual auras are characterized by bright scintillating lights or zigzag lines, often with a scotoma that interferes with reading. Visual auras typically expand over 5 to 20 minutes and last for less than 60 minutes. They are often, but not always restricted to one hemifield. Other types of migraine aura, for example, somatosensory or dysphasic aura, are not included as diagnostic criteria because their phenomenology is less specific and most patients also have visual auras.

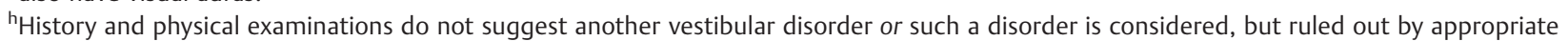
investigations; or such disorder is present as a comorbid or independent condition, but episodes can be clearly differentiated. Migraine attacks may be induced by vestibular stimulation (Murdin et $\mathrm{al}^{23}$ ). Therefore, the differential diagnosis should include other vestibular disorders complicated by superimposed migraine attacks.

Vestibular migraine often misses not only the duration criterion for an aura as defined by the ICHD-2, but also the temporal relationship to migraine headaches: Vertigo can precede headache as would be typical for an aura, may begin with headache, or may appear late in the headache phase. Many patients experience attacks both with and without headache. ${ }^{4,6,7}$ Quite frequently, patients have an attenuated headache with their vertigo as compared with their usual migraine. ${ }^{3,6}$ In some patients, vertigo and headache never occur together. ${ }^{4,6,8}$ Along with the vertigo, patients may experience photophobia, phonophobia, osmophobia, and visual or other auras. These phenomena are of diagnostic importance because they may represent the only apparent connection of vertigo and migraine. Patients need to be asked specifically about these migraine symptoms because they often do not volunteer them. A dizziness diary can be useful for prospective recording of associated features.

Auditory symptoms, including hearing loss, tinnitus, and aural pressure have been reported in up to $38 \%$ patients with vestibular migraine. ${ }^{2,5,6,33,34}$ Hearing loss is usually mild and transient, without or with only minor progression in the course of the disease. ${ }^{6}$ About 20\% develop mild bilateral downsloping hearing loss over the years. ${ }^{35}$ Asking for migraine-specific precipitants of vertigo attacks may provide valuable diagnostic information, for example, provocation by menstruation, deficient sleep, excessive stress, specific foods 
and sensory stimuli, such as bright or scintillating lights, intense smells, or noise. Sometimes, migraine accompaniments and typical precipitants may be missing, but vestibular migraine is still considered the most likely diagnosis after other potential causes have been investigated and appear unlikely. In this case, a favorable response to antimigraine drugs may support the suspicion of an underlying migraine mechanism. However, apparent efficacy of a drug should not be regarded as a definite confirmation of the diagnosis because spontaneous improvement, placebo response, and additional drug effects (e.g., anxiolytic or antidepressant) have to be taken into account.

\section{Clinical and Neuro-Otologic Findings}

In most patients, the general neurologic and otologic examination is normal in the symptom-free interval. ${ }^{4}$ About 10 to $30 \%$ of patients with vestibular migraine have unilateral hypoexcitability to caloric stimulation and $\sim 10 \%$ have directional preponderance of nystagmus responses. ${ }^{4,7,36,37}$ Such findings, however, are not specific for vestibular migraine because they can be found also in migraine patients without vestibular symptoms ${ }^{38}$ and in many other vestibular syndromes. Neuro-ophthalmologic evaluation may reveal mild central deficits such as persistent positional nystagmus and saccadic pursuit, particularly in patients with a long history of vestibular migraine. ${ }^{7,35,39}$ Interictal head-shaking nystagmus was observed in $50 \%$ of vestibular migraine patients. ${ }^{37}$ In one study, patients with vestibular migraine became nauseous after caloric testing four times more often than migraine patients with other vestibular disorders. ${ }^{40}$ Vestibular evoked myogenic potentials (VEMPs) can be abnormal in vestibular migraine, but the findings from various studies are heterogeneous. Abnormalities included reduced electromyographic(EMG-) corrected amplitudes, ${ }^{41}$ unilateral or bilateral loss of VEMP responses, ${ }^{42}$ prolonged latencies, ${ }^{43}$ as well as a shift of the maximum VEMP response from 500 to $1000 \mathrm{~Hz} .^{43}$

A neuro-otologic study of 20 patients during the acute phase of vestibular migraine showed pathological nystagmus in 14 patients, mostly central spontaneous or positional nystagmus. Three patients had a peripheral type of spontaneous nystagmus and a unilateral deficit of the horizontal vestibulo-ocular reflex. Imbalance was observed in all patients except one. ${ }^{44}$ Another study confirmed the high prevalence of persistent positional nystagmus, which was often horizontal and direction-changing, but could also beat in the vertical or torsional plane. ${ }^{45}$

In clinical practice, history will usually provide more clues for the diagnosis than vestibular testing because there are no abnormalities that are specific for vestibular migraine. Therefore, in patients with a clear-cut history, no additional vestibular tests are required. However, vestibular testing in the interval can be useful to reassure patient and doctor that there is no severe abnormality, such as a complete canal paresis that would suggest another diagnosis. Magnetic resonance imaging (MRI) is required in patients presenting with central abnormalities and no previous history of similar attacks. Audiometry helps to differentiate vestibular migraine from Ménière’s disease.

\section{Pathophysiology}

Although a sporadic disorder in most patients, vestibular migraine can be inherited in an autosomal dominant fashion. ${ }^{26}$ A linkage analysis in a four-generation family with 10 affected individuals mapped the locus for vestibular migraine to chromosome $5 q 35 .{ }^{46}$ In a larger study, familial vestibular migraine was found to be genetically heterogenous with a subgroup linking to chromosome $22 \mathrm{q} 12 .{ }^{47}$

The neural mechanisms of vestibular migraine are still obscure. The variability of symptoms and clinical findings both during and between attacks suggests that migraine interacts with the vestibular system at various levels. ${ }^{48}$ Spreading depression, which is the presumed mechanism of the migraine aura, may play a role in patients with short attacks. ${ }^{4}$ Spreading depression is a cortical mechanism that could produce vestibular symptoms when it reaches the multisensory vestibular cortex, which is mainly located in the posterior insula and at the temporoparietal junction. However, several findings during the acute stage of vestibular migraine, including canal paresis and complex positional nystagmus, cannot be explained by cortical dysfunction. $^{42}$

Some of the neurotransmitters that are involved in the pathogenesis of migraine (calcitonin-gene related peptide, serotonin, noradrenaline, dopamine) are also known to modulate the activity of central and peripheral vestibular neurons and could contribute to the pathogenesis of vestibular migraine. ${ }^{4-6,48}$ One may speculate that unilateral release of these substances-in analogy with the often unilateral location of headaches-causes a static vestibular imbalance leading to rotatory vertigo, whereas bilateral release would induce a state of altered vestibular excitability leading to a motion sickness type of dizziness. Plasma extravasation from dural vessels causing transient meningeal inflammation is considered to be a key mechanism in migraine. In mice, serotonin-induced plasma extravasation was observed not only in the dura mater, but also in the inner ear. ${ }^{49}$ Vestibular and nociceptive pathways show neurochemical similarities and share central pathways for perception, interoception, and affect. $^{48}$

Genetic defects of a voltage-gated calcium channel have been identified as the cause of familial hemiplegic migraine and episodic ataxia type 2 (EA-2). ${ }^{50}$ As both of these paroxysmal disorders can have vertigo and migraine headache as prominent symptoms, a defective gene in the same region is a candidate mechanism for vestibular migraine. However, so far no such genetic defect has been identified. . $^{51,52}$

The only hypothesis that is actually based on a human experimental model of vestibular migraine relates to the known reciprocal connections between the trigeminal and vestibular nuclei. Trigeminal activation by painful electrical stimulation of the forehead produced spontaneous nystagmus in migraine patients, but not in controls, indicating that those with migraine have a lowered threshold for crosstalk between these neighboring brainstem structures. $^{53}$ 
Table 2 Prophylactic treatment of vestibular migraine

\begin{tabular}{|l|l|l|}
\hline Drug & Daily dose & Side effects \\
\hline Propranolol (Harker \& Rassekh, 198756) & $40-240 \mathrm{mg}$ & $\begin{array}{l}\text { Fatigue, hypotension, impotence, depression, } \\
\text { nightmares, bronchial constriction }\end{array}$ \\
\hline Metoprolol (Dieterich \& Brandt, 19997) & $50-200 \mathrm{mg}$ & $\begin{array}{l}\text { Fatigue, hypotension, impotence, depression, } \\
\text { nightmares, bronchial constriction }\end{array}$ \\
\hline Amitriptyline (Reploeg \& Goebel, 2002 ${ }^{9}$ ) & $50-100 \mathrm{mg}$ & $\begin{array}{l}\text { Sedation, orthostatic hypotension, dry mouth, } \\
\text { weight gain, constipation, urinary retention, } \\
\text { conduction block }\end{array}$ \\
\hline Flunarizine (Dieterich \& Brandt, 19997) & $5-10 \mathrm{mg}$ & $\begin{array}{l}\text { Weight gain, sedation, depression, reversible } \\
\text { parkinsonism }\end{array}$ \\
\hline Acetazolamide (Baloh et al, 1996 ${ }^{57}$ ) & $250-750 \mathrm{mg}$ & $\begin{array}{l}\text { Paresthesia, nausea, sedation, hypokalemia, } \\
\text { hyperglycemia }\end{array}$ \\
\hline
\end{tabular}

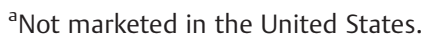

\section{Treatment}

In many patients, vestibular migraine attacks are severe, long, and frequent enough to warrant acute or prophylactic treatment. Unfortunately, current treatment recommendations are based on expert opinion rather than on solid data from randomized placebo-controlled trials. And indeed, apart from one small and inconclusive study on the use of zolmitriptan for acute vestibular migraine, no proper study has been undertaken. ${ }^{54}$ A review of treatment trials identified eight retrospective studies that showed marginal improvement with migraine prophylactic medications. ${ }^{55}$ In the absence of firm evidence, no single prophylactic agent appears to be preferable. Expected side effects, such as orthostatic hypotension with beta blockers or weight gain with valproate or amitriptyline, will influence the selection of the drug (-Table 2). Patients should monitor frequency and severity of their attacks in a diary. Treatment response should be evaluated after 3 months. A greater than $50 \%$ reduction in attack frequency is a reasonable goal. The carboanhydrase inhibitors acetazolamide and dichlorphenamide, which are not normally used for migraine prophylaxis, appeared effective in retrospective case series. $^{57,58}$ For acute attacks, conventional antiemetic drugs such as dimenhydrinate or benzodiazepines seem most appropriate. Intravenous methylprednisolone $(1000 \mathrm{mg} / \mathrm{d}$ for 1 $3 \mathrm{~d}$ ) effectively terminated prolonged attacks or exacerbations with daily recurrences in four patients. ${ }^{59}$

Nonpharmaceutical approaches in the treatment of vestibular migraine should not be neglected and may be even more effective than drugs in individual patients. A thorough explanation of the migraine origin of the attacks can relieve unnecessary fears. Avoidance of identified triggers, regular sleep, regular meals, and exercise has a firm place in migraine prophylaxis. Selected patients, particularly those with persistent symptoms between attacks, may profit from vestibular rehabilitation. ${ }^{60}$

\section{Migraine and Ménière's Disease}

Migraine is more common in patients with Ménière's disease than in healthy controls. ${ }^{61}$ Patients with features of both Ménière's disease and vestibular migraine have been repeatedly reported. 34,61,62 In fact, migraine and Ménière's disease can be inherited as a symptom cluster. ${ }^{63}$ Fluctuating hearing loss, tinnitus, and aural pressure may occur in vestibular migraine, but hearing loss does not progress to profound levels. ${ }^{6,35}$ Similarly, migraine headaches, photophobia, and even migraine auras are common during Ménière attacks. ${ }^{61,64}$ The pathophysiological relationship between vestibular migraine and Ménière's disease remains uncertain. In the first few years after onset of symptoms, differentiation of vestibular migraine from Ménière's disease may be challenging, as Ménière's disease can be monosymptomatic with vestibular symptoms only in the early stages of the disease. ${ }^{65}$

\section{Migraine Associated with Other Disorders Causing Dizziness and Vertigo}

There is a more than chance association of migraine with several other established vertigo and dizziness syndromes including benign paroxysmal positional vertigo, ${ }^{66}$ motion sickness, ${ }^{67}$ orthostatic hypotension and syncope, ${ }^{68}$ panic disorder, ${ }^{69}$ and depression. ${ }^{70}$ These disorders may be the only cause of vestibular symptoms in a patient with migraine or may coexist with vestibular migraine. Patients with vestibular migraine show the highest rate of concurrent anxiety or depressive disorders as compared with other vertigo syndromes. ${ }^{71}$ Because of the frequent association of dizziness, migraine, and anxiety, a new syndrome named migraine-anxiety-related dizziness (MARD) has been proposed. ${ }^{72}$

\section{References}

1 Liveing E. On megrim: Sick headache and some allied health disorders: a contribution to the pathology of nerve storms. London: Churchill; 1873:129-148

2 Kayan A, Hood JD. Neuro-otological manifestations of migraine. Brain 1984;107(Pt 4):1123-1142

3 Behan PO, Carlin J. Benign recurrent vertigo. New York: Raven Press; 1982 
4 Cutrer FM, Baloh RW. Migraine-associated dizziness. Headache 1992;32(6):300-304

5 Cass SP, Furman JM, Ankerstjerne K, Balaban C, Yetiser S, Aydogan B. Migraine-related vestibulopathy. Ann Otol Rhinol Laryngol 1997;106(3):182-189

6 Johnson GD. Medical management of migraine-related dizziness and vertigo. Laryngoscope 1998;108(1 Pt 2):1-28

7 Dieterich M, Brandt T. Episodic vertigo related to migraine (90 cases): vestibular migraine? J Neurol 1999;246(10):883-892

8 Neuhauser H, Leopold M, von Brevern M, Arnold G, Lempert T. The interrelations of migraine, vertigo, and migrainous vertigo. Neurology 2001;56(4):436-441

9 Reploeg MD, Goebel JA. Migraine-associated dizziness: patient characteristics and management options. Otol Neurotol 2002;23 (3):364-371

10 Brandt T, Strupp M. Migraine and vertigo: classification, clinical features, and special treatment considerations. Headache Currents 2006;3:12-19

11 Lempert T, Olesen J, Furman J, et al. Vestibular migraine: diagnostic criteria. J Vestib Res 2012;22(4):167-172

12 International Headache Society Classification Subcommittee. International classification of headache disorders. 2nd edition. Cephalalgia 2004;24(Suppl 1):1-160

13 Lee H, Sohn SI, Jung DK, et al. Migraine and isolated recurrent vertigo of unknown cause. Neurol Res 2002;24(7):663-665

14 Cha YH, Lee H, Santell LS, Baloh RW. Association of benign recurrent vertigo and migraine in 208 patients. Cephalalgia 2009;29(5):550-555

15 Kuritzky A, Ziegler DK, Hassanein R. Vertigo, motion sickness and migraine. Headache 1981;21(5):227-231

16 Vuković V, Plavec D, Galinović I, Lovrencić-Huzjan A, Budisić M, Demarin V. Prevalence of vertigo, dizziness, and migrainous vertigo in patients with migraine. Headache 2007;47(10):14271435

17 Jensen R, Stovner LJ. Epidemiology and comorbidity of headache. Lancet Neurol 2008;7(4):354-361

18 Neuhauser HK, von Brevern M, Radtke A, et al. Epidemiology of vestibular vertigo: a neurotologic survey of the general population. Neurology 2005;65(6):898-904

19 Neuhauser HK, Radtke A, von Brevern M, et al. Migrainous vertigo: prevalence and impact on quality of life. Neurology 2006;67 (6):1028-1033

20 Sturzenegger $\mathrm{MH}$, Meienberg O. Basilar artery migraine: a followup study of 82 cases. Headache 1985;25(8):408-415

21 Bisdorff A, Von Brevern M, Lempert T, Newman-Toker DE. Classification of vestibular symptoms: towards an international classification of vestibular disorders. J Vestib Res 2009;19(1-2):1-13

22 Versino M, Sances G, Anghileri E, et al. Dizziness and migraine: a causal relationship? Funct Neurol 2003;18(2):97-101

23 Murdin L, Davies RA, Bronstein AM. Vertigo as a migraine trigger. Neurology 2009;73(8):638-642

24 Hsu LC, Wang SJ, Fuh JL. Prevalence and impact of migrainous vertigo in mid-life women: a community-based study. Cephalalgia 2011;31(1):77-83

25 Geser R, Straumann D. Referral and final diagnoses of patients assessed in an academic vertigo center. Frontiers Neurol 2012;3: Article 169

26 Oh AK, Lee H, Jen JC, Corona S, Jacobson KM, Baloh RW. Familial benign recurrent vertigo. Am J Med Genet 2001;100(4):287-291

27 Basser LS. Benign paroxysmal vertigo of childhood (a variety of vestibular neuronitis). Brain 1964;87:141-152

28 Krams B, Echenne B, Leydet J, Rivier F, Roubertie A. Benign paroxysmal vertigo of childhood: long-term outcome. Cephalalgia 2011;31(4):439-443

29 Abu-Arafeh I, Russell G. Paroxysmal vertigo as a migraine equivalent in children: a population-based study. Cephalalgia 1995;15 (1):22-25, discussion 4
30 Slater R. Benign recurrent vertigo. J Neurol Neurosurg Psychiatry 1979;42(4):363-367

31 von Brevern M, Radtke A, Clarke AH, Lempert T. Migrainous vertigo presenting as episodic positional vertigo. Neurology 2004;62 (3):469-472

32 Waterston J. Chronic migrainous vertigo. J Clin Neurosci 2004;11 (4):384-388

33 Parker W. Migraine and the vestibular system in adults. Am J Otol 1991;12(1):25-34

34 Neff BA, Staab JP, Eggers SD, et al. Auditory and vestibular symptoms and chronic subjective dizziness in patients with Ménière's disease, vestibular migraine, and Ménière's disease with concomitant vestibular migraine. Otol Neurotol 2012;33 (7):1235-1244

35 Radtke A, von Brevern M, Neuhauser H, Hottenrott T, Lempert T. Vestibular migraine: long-term follow-up of clinical symptoms and vestibulo-cochlear findings. Neurology 2012;79(15):16071614

36 Celebisoy N, Gökçay F, Sirin H, Biçak N. Migrainous vertigo: clinical, oculographic and posturographic findings. Cephalalgia 2008;28 (1):72-77

37 Shin JE, Kim CH, Park HJ. Vestibular abnormality in patients with Meniere's disease and migrainous vertigo. Acta Otolaryngol 2013;133(2):154-158

38 Harno H, Hirvonen T, Kaunisto MA, et al. Subclinical vestibulocerebellar dysfunction in migraine with and without aura. Neurology 2003;61(12):1748-1752

39 Neugebauer H, Adrion C, Glaser M, Strupp M. Long-term changes of central ocular motor signs in patients with vestibular migraine. Eur Neurol 2013;69(2):102-107

40 Vitkovic J, Paine M, Rance G. Neuro-otological findings in patients with migraine- and nonmigraine-related dizziness. Audiol Neurootol 2008;13(2):113-122

41 Baier B, Stieber N, Dieterich M. Vestibular-evoked myogenic potentials in vestibular migraine. J Neurol 2009;256(9):14471454

42 Boldingh MI, Ljøstad U, Mygland A, Monstad P. Vestibular sensitivity in vestibular migraine: VEMPs and motion sickness susceptibility. Cephalalgia 2011;31(11):1211-1219

43 Murofushi T, Ozeki H, Inoue A, Sakata A. Does migraine-associated vertigo share a common pathophysiology with Meniere's disease? Study with vestibular-evoked myogenic potential. Cephalalgia 2009;29(12):1259-1266

44 von Brevern M, Zeise D, Neuhauser H, Clarke AH, Lempert T. Acute migrainous vertigo: clinical and oculographic findings. Brain 2005;128(Pt 2):365-374

45 Polensek SH, Tusa RJ. Nystagmus during attacks of vestibular migraine: an aid in diagnosis. Audiol Neurootol 2010;15(4): 241-246

46 Bahmad F Jr, DePalma SR, Merchant SN, et al. Locus for familial migrainous vertigo disease maps to chromosome $5 q 35$. Ann Otol Rhinol Laryngol 2009;118(9):670-676

47 Lee H, Jen JC, Wang H, et al. A genome-wide linkage scan of familial benign recurrent vertigo: linkage to 22q12 with evidence of heterogeneity. Hum Mol Genet 2006;15(2):251-258

48 Balaban CD. Migraine, vertigo and migrainous vertigo: links between vestibular and pain mechanisms. J Vestib Res 2011;21 (6):315-321

49 Koo JW, Balaban CD. Serotonin-induced plasma extravasation in the murine inner ear: possible mechanism of migraine-associated inner ear dysfunction. Cephalalgia 2006;26(11):1310-1319

50 Ophoff RA, Terwindt GM, Vergouwe MN, et al. Familial hemiplegic migraine and episodic ataxia type- 2 are caused by mutations in the Ca2 + channel gene CACNL1A4. Cell 1996;87(3):543-552

51 Kim JS, Yue Q Jen JC, Nelson SF, Baloh RW. Familial migraine with vertigo: no mutations found in CACNA1A. Am J Med Genet 1998;79(2):148-151 
52 von Brevern M, Ta N, Shankar A, et al. Migrainous vertigo: mutation analysis of the candidate genes CACNA1A, ATP1A2, SCN1A, and CACNB4. Headache 2006;46(7):1136-1141

53 Marano E, Marcelli V, Di Stasio E, et al. Trigeminal stimulation elicits a peripheral vestibular imbalance in migraine patients. Headache 2005;45(4):325-331

54 Neuhauser H, Radtke A, von Brevern M, Lempert T. Zolmitriptan for treatment of migrainous vertigo: a pilot randomized placebocontrolled trial. Neurology 2003;60(5):882-883

55 Fotuhi M, Glaun B, Quan SY, Sofare T. Vestibular migraine: a critical review of treatment trials. J Neurol 2009;256(5):711-716

56 Harker LA, Rassekh CH. Episodic vertigo in basilar artery migraine. Otolaryngol Head Neck Surg 1987;96:239-250

57 Baloh RW, Foster CA, Yue Q Nelson SF. Familial migraine with vertigo and essential tremor. Neurology 1996;46(2):458-460

58 Asprella Libonati G, Gagliardi G. La malattia di Meniere e vertigine emicranica: terapi intercritica, terapia medica. Otoneurologia 2004; 18:40-42

59 Prakash S, Shah ND. Migrainous vertigo responsive to intravenous methylprednisolone: case reports. Headache 2009;49(8): 1235-1239

60 Whitney SL, Wrisley DM, Brown KE, Furman JM. Physical therapy for migraine-related vestibulopathy and vestibular dysfunction with history of migraine. Laryngoscope 2000;110(9):1528-1534

61 Radtke A, Lempert T, Gresty MA, Brookes GB, Bronstein AM, Neuhauser $\mathrm{H}$. Migraine and Ménière's disease: is there a link? Neurology 2002;59(11):1700-1704

62 Cha YH, Brodsky J, Ishiyama G, Sabatti C, Baloh RW. The relevance of migraine in patients with Meńière's disease. Acta Otolaryngol 2007;127(12):1241-1245
63 Cha YH, Kane MJ, Baloh RW. Familial clustering of migraine, episodic vertigo, and Ménière's disease. Otol Neurotol 2008;29 (1):93-96

64 Brantberg K, Baloh RW. Similarity of vertigo attacks due to Meniere's disease and benign recurrent vertigo, both with and without migraine. Acta Otolaryngol 2011;131(7):722-727

65 Pyykkö I, Nakashima T, Yoshida T, Zou J, Naganawa S. Meniere's disease: a reappraisal supported by a variable latency of symptoms and the MRI visualisation of endolymphatic hydrops. BMJ Open 2013;3(2):e001555

66 Ishiyama A, Jacobson KM, Baloh RW. Migraine and benign positional vertigo. Ann Otol Rhinol Laryngol 2000;109(4):377-380

67 Jeong SH, Oh SY, Kim HJ, Koo JW, Kim JS. Vestibular dysfunction in migraine: effects of associated vertigo and motion sickness. J Neurol 2010;257(6):905-912

68 Thijs RD, Kruit MC, van Buchem MA, Ferrari MD, Launer LJ, van Dijk JG. Syncope in migraine: the population-based CAMERA study. Neurology 2006;66(7):1034-1037

69 Breslau N, Schultz LR, Stewart WF, Lipton RB, Welch KM. Headache types and panic disorder: directionality and specificity. Neurology 2001;56(3):350-354

70 Breslau NL, Schultz LR, Stewart WF, Lipton RB, Lucia VC, Welch KM. Headache and major depression: is the association specific to migraine? Neurology 2000;54(2):308-313

71 Eckhardt-Henn A, Best C, Bense S, et al. Psychiatric comorbidity in different organic vertigo syndromes. J Neurol 2008;255(3):420428

72 Furman JM, Balaban CD, Jacob RG, Marcus DA. Migraine-anxiety related dizziness (MARD): a new disorder? J Neurol Neurosurg Psychiatry 2005;76(1):1-8 